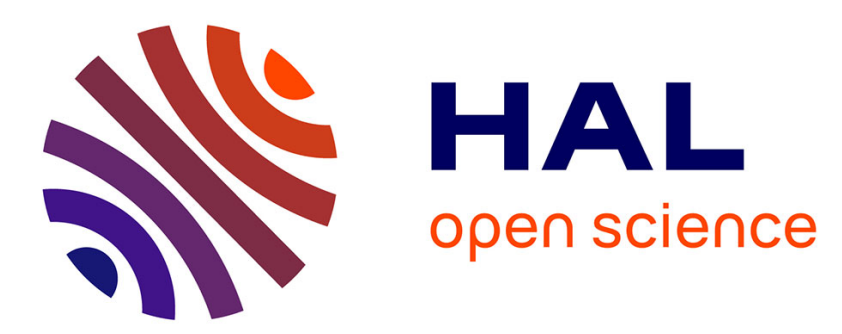

\title{
Les ethnothéories de l'éducation, quel accord entre parents migrants et enseignantes?
}

\author{
Xavier Conus, Tania Ogay
}

\section{To cite this version:}

Xavier Conus, Tania Ogay. Les ethnothéories de l'éducation, quel accord entre parents migrants et enseignantes? . Revue internationale de l'éducation familiale, 2014, 35, pp.95-123. 10.3917/rief.035.0093 . hal-01256873

\section{HAL Id: hal-01256873 https://hal.science/hal-01256873}

Submitted on 15 Jan 2016

HAL is a multi-disciplinary open access archive for the deposit and dissemination of scientific research documents, whether they are published or not. The documents may come from teaching and research institutions in France or abroad, or from public or private research centers.
L'archive ouverte pluridisciplinaire HAL, est destinée au dépôt et à la diffusion de documents scientifiques de niveau recherche, publiés ou non, émanant des établissements d'enseignement et de recherche français ou étrangers, des laboratoires publics ou privés. 
Citer comme: Conus, X. \& Ogay, T. (2014). Les ethnothéories de l'éducation, quel accord entre parents migrants et enseignantes ? Revue internationale d'éducation familiale, 35, 95-123. doi : 10.3917/rief.035.0093

\section{Les ethnothéories de l'éducation, quel accord entre parents migrants et enseignantes ?}

\section{Ethnotheories of education, which agreement between migrant parents and teachers?}

\section{Xavier CONUS ${ }^{1}$ \\ Tania OGAY ${ }^{1}$}

\section{Résumé}

En prologue à une recherche portant sur la construction de la relation familles-école au moment de l'entrée à l'école, nous présentons ici les résultats d'une recherche préliminaire, descriptive et quantitative, dont l'objectif est de comparer parents migrants et enseignants du point de vue de leurs conceptions (ou "ethnothéories" selon le modèle de la niche développementale) de l'éducation et du développement de l'enfant. L'enquête a été réalisée au moyen d'un questionnaire auquel ont répondu 31 enseignantes de l'école enfantine, 27 parents migrants portugais et 22 parents migrants africains, tous résidant à Fribourg (Suisse). Si les résultats témoignent de différences intergroupes, ils montrent surtout une divergence entre les parents et les enseignantes, ce qui interroge par rapport à une relation de partenariat souvent présentée comme allant de soi.

Mots-clés : Ethnothéories, enseignants, parents migrants, coéducation, petite enfance, interculturalité.

\section{Abstract}

The research presented in this article is a preliminary study of a larger project about the construction of the relation between families and school at school entry. The objective of the descriptive and quantitative preliminary study is to compare migrant parents and teachers in their conceptions (or "ethnotheories" in the model of the developmental niche) of child development and education. The data were collected by means of

\footnotetext{
${ }^{1}$ Université de Fribourg, Département des Sciences de l'éducation, rue de Faucigny 2, CH-1700 Fribourg.Xavier.Conus@unifr.ch
} 
a questionnaire answered by 31 kindergarten teachers, 27 Portuguese migrant parents and 22 African migrant parents, all living in Fribourg (Switzerland). If results do show intergroup differences, they above all show a divergence between the parents and the teachers. This brings to consider with precaution a relation of partnership which is often presented as being self-evident.

Keywords : Ethnotheories, teachers, migrant parents, coeducation, early childhood, interculturality.

« Entre parents et enseignants : un dialogue impossible ? (Montandon et Perrenoud, 1994), «L'école et la famille, deux mondes ?» (Claes et Comeau, 1996), «L'école contre les parents » (Gayet, 1999), «Ecole, familles: le malentendu » (Dubet, 2001). Au-delà des titres peut-être accrocheurs, un constat s'impose : la relation entre l'école et les familles est souvent difficile, en particulier pour les familles minoritaires, plus éloignées de la culture scolaire (Kim, 2009). La recherche ayant établi un lien entre la qualité de la relation familles-école et la réussite scolaire des élèves (Comer, 1988; Epstein, 1991; Reynolds, 1992; Epstein et Sanders, 2002; Porumbu et Necşoi, 2013), l'intérêt des chercheurs et responsables des systèmes éducatifs se porte en particulier sur les familles migrantes et/ou «populaires », leurs enfants étant plus souvent en échec scolaire (Field, Kuczera et Pont, 2007). Faisant écho au reproche récurrent de démission formulé par les enseignants à l'égard de ces parents (Périer, 2005), les chercheurs se sont intéressés à l'engagement parental dans la scolarité des enfants (Poncelet et Francis, 2010), identifiant les obstacles et facilitateurs (notamment Hoover-Dempsey et Sandler, 1997).

Cependant, comme le relève Epstein (2011), le concept d'engagement parental occulte la responsabilité partagée des trois sphères d'influence engagées dans le développement de l'enfant - la famille, l'école et la communauté -, toute la responsabilité de la relation défaillante semble être mise sur les parents (les «familles en faute», selon Périer, 2005). Epstein (2011) préfère parler de partenariat, à comprendre dans une vision écologique et systémique. Dans le champ de la petite enfance, il est beaucoup question de coéducation (Mackiewicz, 2010; Rayna, 2010). Celle-ci met en avant la contribution de chacun, parents et professionnels, qui ont à conjuguer leurs compétences afin de réaliser l'objectif commun du développement harmonieux de l'enfant :

« Les principes de la coéducation sont donc à rapprocher de ceux de cohérence éducative entre les milieux de vie de l'enfant. Pour que l'enfant évolue dans un environnement éducatif cohérent, il est nécessaire que les différents acteurs de son éducation se côtoient. Cette condition est nécessaire mais insuffisante. Il faut 
aussi que les uns et les autres se reconnaissent des compétences, acceptent et puissent modifier leurs stratégies et pratiques en conséquence, par enrichissement mutuel » (Prévôt, 2008, p. 39).

Se reconnaître comme co-éducateurs implique qu'il y ait un échange, un partage sur les façons d'éduquer, même si cela n'implique pas nécessairement une uniformité. Les conceptions - ou ethnothéories selon le modèle de la niche développementale (Super et Harkness, 1986, 1996) - des parents et professionnels au sujet de l'éducation de l'enfant et de leurs rôles respectifs ainsi que la négociation de ces conceptions se trouvent au cour du partenariat et, a fortiori, de la coéducation. La réussite scolaire de l'enfant peut même être en jeu : dans la recherche de Hauser-Cram, Sirin et Stipek (2003), les enseignants qui percevaient des différences entre les valeurs éducatives des parents et les leurs voyaient les élèves comme moins compétents.

Nous avons choisi de nous intéresser aux conceptions de l'éducation et à leur négociation au moment de la genèse de la relation familles-école : lors de l'entrée à l'école, moment-clé de transition pour l'enfant et ses parents qui voient leur contexte écologique transformé par l'arrivée de ce nouvel acteur (Rimm-Kaufman et Pianta, 2000). La recherche que nous présentons ici s'inscrit dans un projet plus large, encore en phase pilote, dans lequel nous cherchons à saisir comment se construit la relation entre les familles et l'école lors de la première année de scolarité2, en étudiant en particulier les interactions entre enseignantes et parents. Dans une démarche ethnographique, nous combinons observations et entretiens auprès des familles et enseignantes des quatre classes d'école enfantine d'un établissement scolaire, situé dans un quartier péri-urbain de Fribourg (Suisse), accueillant une population souvent immigrée et aux revenus modestes.

La démarche de la recherche que nous rapportons ici, quantitative, descriptive et comparative (Conus, 2012), se situe en amont de celle du projet. Avant d'étudier comment se négocient les conceptions de l'éducation dans les interactions entre parents et enseignantes, nous voulions repérer quelles peuvent être ces différentes conceptions en cherchant à voir le rôle de la variable culturelle. Dans cette optique, nous avons investigué les conceptions de l'éducation de parents migrants venus du Portugal et d'Afrique subsaharienne ainsi que d'enseignantes d'école enfantine. L'identification des différentes conceptions de l'éducation vise à une meilleure compréhension des cadres de références

\footnotetext{
${ }^{2}$ Dans la plupart des cantons suisses, l'entrée à l'école est fixée depuis peu à 4 ans révolus. Dans le canton de Fribourg, les deux premières années d'école sont encore appelées "école enfantine".
} 
réciproques, nous situant au niveau des deux premières étapes de l'approche interculturelle de Cohen-Emerique (2011), la décentration de son propre cadre de références et la découverte du cadre de références de l'autre. La troisième étape de l'approche de Cohen-Emerique, celle de la négociation, sera l'objet de la suite de la recherche.

\section{Des conceptions aux ethnothéories de l'éducation}

"Conceptions", “croyances", "représentations", "théories naïves”... Parmi les divers termes utilisés en sciences sociales pour désigner les idées que se font les individus ou les groupes sociaux d'un objet, nous privilégions celui d'ethnothéories, emprunté au modèle théorique de la niche développementale (Super et Harkness, 1986, 1996). Selon ces auteurs, les ethnothéories de l'éducation et du développement sont les représentations mentales, les théories de sens commun (même si leurs fondements sont parfois scientifiques), à la fois formelles et informelles, conscientes ou non conscientes, qui touchent à l'éducation de l'enfant et à son développement. Elles forment un cadre de référence, servant à l'interprétation de la réalité et des événements comme à la mise en œuvre des conduites et actions pour tout ce qui concerne l'éducation et le développement de l'enfant. Les ethnothéories de l'éducation sont l'une des trois composantes de la niche développementale de l'enfant, avec les pratiques éducatives des «caretakers» (parents, enseignants, etc.) et les contextes physiques et sociaux autour de l'enfant. Ces trois composantes sont reliées et s'influencent réciproquement de façon systémique, avec des mécanismes visant à un équilibre, dans un système où l'individu luimême (l'enfant, mais aussi le « caretaker ») est à la fois influencé par le système et l'influence en retour (Bowler, 2005).

Dans son cadre théorique intégré du développement humain, Dasen (2004) reprend le modèle de la niche développementale de Super et Harkness, qu'il complexifie en l'articulant avec le modèle écosystémique de Bronfenbrenner (1979) et avec le cadre théorique écoculturel de Berry (Berry, Poortinga, Segall et Dasen, 2002). La niche développementale correspond au microsystème de Bronfenbrenner. Elle est influencée par le macrosystème (contextes écologique et socio-politique, valeurs, cosmologies, religions, adaptation biologique et culturelle). Le mésosystème fait le lien entre les niveaux macro- et micro-, par le biais des processus mis en avant dans le cadre théorique écoculturel de Berry, à savoir les processus d'influences écologiques, de transmission génétique, de transmission culturelle et d'acculturation. Ces deux derniers processus sont particulièrement pertinents pour nos deux groupes de répondants migrants: le processus de transmission culturelle, par lequel les membres acquièrent la culture du groupe (toujours de manière en 
partie individuelle), et le processus d'acculturation, qui résulte du contact avec une autre culture et des changements culturels qui en découlent, processus dont l'influence sur les ethnothéories ne doit donc pas être négligée dans la situation de parents migrants. La constitution des ethnothéories résulte ainsi d'un jeu d'influences multiples, intervenant à tous les niveaux de l'écosystème dans lequel s'inscrit la niche développementale de l'enfant.

La complexité du modèle de Dasen souligne que les ethnothéories se construisent selon un processus actif, en lien avec la culture, l'histoire et sa propre éducation. Les ethnothéories font partie de la culture et témoignent de la même complexité, elles sont également dynamiques, processuelles et évolutives. Si les ethnothéories de l'individu sont en partie tributaires de dimensions collectives, en lien avec ses groupes culturels d'appartenance, elles revêtent également une dimension individuelle, à la fois par l'histoire personnelle, par les différences existant entre membres d'un même groupe et par l'imbrication toujours particulière de ses différents groupes d'appartenance. Cette dimension individuelle des ethnothéories fait que les membres d'un même groupe ne partagent jamais exactement les mêmes ethnothéories. Par notre approche comparative intergroupes, nous avons choisi de nous intéresser davantage à la dimension collective des ethnothéories, mais en veillant à ne pas ignorer la dimension individuelle et la variabilité intragroupe. Ceci s'est traduit par la prise en compte également de variables intragroupes dans l'analyse.

En portant plus spécifiquement notre attention sur les ethnothéories de parents migrants venus du Portugal et d'Afrique subsaharienne pour s'installer à Fribourg (Suisse), nous avons voulu investiguer l'influence de la variable culturelle sur les ethnothéories de groupes de sujets très présents dans le contexte local et dont on peut supposer qu'ils ont des références culturelles différentes de celles des enseignants de l'école fribourgeoise (ceux-ci, en plus de partager une même culture professionnelle, étant un groupe de population très homogène). Le choix de regrouper les parents d'Afrique subsaharienne dans un même groupe se justifie par les travaux de différents chercheurs relevant un certain nombre de dimensions transversales et partagées permettant de parler d'une conception africaine de l'éducation (Dasen, 2002 ; Erny, 1987, 2001 ; Nsamenang, 2001, 2005). Quant aux parents migrants portugais, l'intérêt que nous leur portons est double : construire l'étude comparative avec deux groupes de parents migrants considérés, dans l'opinion commune, comme ayant des références culturelles différentes de celles de la société d'accueil ; avec les parents migrants portugais, s'intéresser aux conceptions de l'éducation d'un groupe souvent pointé du doigt pour les difficultés scolaires rencontrées par ses enfants (voir notamment Doudin, 
Lafortune, Pons et Moreau, 2009). Finalement, le modèle de la niche développementale et le concept d'ethnothéories ne sont pas applicables uniquement à des groupes culturels vus comme des groupes ethniques ou nationaux, mais aussi à des groupes partageant une culture professionnelle, comme l'illustre une recherche menée sur les ethnothéories professionnelles de personnel de santé (Leanza, 2003). Nous considérons ainsi que les enseignantes de l'école enfantine fribourgeoise peuvent être considérées comme un groupe culturel, dont les membres partagent des ethnothéories liées à différentes appartenances communes (formation professionnelle, milieu social, lieu d'enculturation) et aux processus de transmission culturelle qui y sont liés.

\section{Méthode}

L'échantillon de l'étude se compose de 31 enseignantes d'école enfantine du canton de Fribourg (Suisse), de 27 parents migrants portugais et de 22 parents migrants africains, tous habitant le canton de Fribourg. Le contact avec les parents s'est fait par l'intermédiaire d'associations communautaires ou institutions travaillant avec des parents migrants. Les enseignantes (aucun répondant masculin sur les 86 professionnels sollicités) ont comme caractéristique d'œuvrer dans des établissements marqués par une diversité des nationalités des élèves, qui viennent souvent de familles aux revenus modestes. Elles sont toutes Suissesses, l'une étant double nationale. Le groupe des parents migrants portugais comporte 27 répondants (4 questionnaires ont été remplis par le père, 20 par la mère, et 3 par les deux parents, malgré les consignes demandant de le remplir de manière individuelle). Le niveau de formation est très homogène: à l'exception de 3 parents ayant suivi une formation secondaire supérieure, les 24 autres ont une formation ne dépassant pas le secondaire inférieur ou l'école obligatoire. L'ancienneté d'arrivée en Suisse va de 2 ans à 22 ans. Le groupe des parents migrants africains compte 22 répondants ( 15 mères, 7 pères). Le niveau de formation est divers, allant d'une scolarisation primaire (7) à une formation universitaire (6). Concernant le pays de départ de la trajectoire migratoire, ces parents viennent de République Démocratique du Congo (7), du Cameroun (4), d'Erythrée (3), du Sénégal (2), du Rwanda (2), du Gabon (1), du Tchad (1), d'Angola (1) et de Somalie (1). L'ancienneté d'arrivée en Suisse va de 3 ans à 20 ans. Les parents de l'échantillon ne sont pas reliés aux enseignantes : leurs enfants ne sont probablement pas scolarisés dans les classes des enseignantes interrogées. Si cela devait être le cas, ce serait dû au hasard et nous n'en avons nulle connaissance.

Le recueil de données s'est fait par un questionnaire ad hoc dont les résultats ont été traités avec une méthodologie d'analyse quantitative, 
aboutissant à une cartographie des ethnothéories chez les parents et enseignantes interrogés. D'autres variables que celle du groupe culturel sont également analysées: l'âge, la formation suivie (école normale ou $\mathrm{HEP}^{3}$ ), le fait d'être parent ou non chez les enseignants, ainsi que le niveau de formation, le type de profession, le sexe, l'ancienneté d'arrivée en Suisse, le nombre d'enfants et le rang de l'enfant dans la fratrie chez les parents.

La construction du questionnaire s'est faite à partir d'une revue non exhaustive de littérature sur les conceptions de l'éducation dans l'éducation traditionnelle africaine (Dasen, 2002; Erny, 1987, 2001; Nsamenang, 2001, 2005) et occidentale (Arm-Spring et al., 2008; Dupeyron, 2010). Cette revue a mis en évidence l'existence de différences culturelles quant aux conceptions de l'éducation de l'enfant. Le questionnaire se compose de 82 items proposant des affirmations sur le développement et l'éducation de l'enfant, en précisant que les items concernent l'éducation d'un enfant de 5 ans, soit en âge de fréquenter l'école enfantine. Certaines affirmations traduisent plutôt des conceptions propres à l'éducation traditionnelle occidentale, d'autres des conceptions relevées dans l'éducation traditionnelle africaine, d'autres enfin reprennent des éléments identifiés dans les deux axes de la revue de littérature. Le répondant est invité à exprimer son degré d'accord sur une échelle de Likert à 5 niveaux («pas du tout d'accord » $=1 /$ « tout à fait d'accord »=5). Pour l'analyse, 11 items ont été supprimés en raison d'effets d'asymétrie (valeurs dépassant 1.96 pour les coefficients de Skewness et de Kurtosis). Les items recouvrent 19 catégories thématiques concernant des domaines de l'éducation de l'enfant, des aspects de son développement, les visions de l'éducation en tant que «caretaker », les stratégies éducatives ou les rôles éducatifs. Le tableau ci-dessous (Tableau 1) détaille les 19 catégories du questionnaire, le nombre d'items et l'alpha de Cronbach correspondant à chaque catégorie.

Tableau 1 : Catégories thématiques du questionnaire

\begin{tabular}{|l|c|c|}
\hline \multicolumn{1}{|c|}{ Catégories thématiques du questionnaire } & Items & $\begin{array}{c}\text { Alpha } \\
\text { Cronbach }\end{array}$ \\
\hline Stratégies éducatives : modèle instructionnel vs. participatif & 4 & .81 \\
\hline Importance de la période du questionnement chez l'enfant & 4 & .61 \\
\hline Milieu éducatif familial stimulant vs. naturel & 4 & .63 \\
\hline
\end{tabular}

${ }^{3}$ Haute Ecole Pédagogique : institution de formation des enseignants en Suisse ayant succédé à l'Ecole normale dans les années 2000 et qui se caractérise, contrairement à l'ancien système, par l'exigence de l'obtention d'une maturité fédérale (baccalauréat) comme condition d'entrée. 


\begin{tabular}{|l|c|c|}
\hline Représentation de l'usage de la récompense & 3 & .82 \\
\hline Représentation de l'usage de la sanction & 4 & .73 \\
\hline Attitudes attendues de l'enfant : maîtrise vs. spontanéité & 5 & .74 \\
\hline Respect envers l'adulte : souple vs. rigide & 4 & .54 \\
\hline Rôle développemental de la situation d'échec & 3 & .54 \\
\hline Rôles éducatifs familiaux : dyade enfant-parents vs. famille élargie & 5 & .80 \\
\hline Rôles éducatifs père-mère : différenciés vs. similaires & 3 & .79 \\
\hline Buts de l'éducation : intégration sociale vs. réussite individuelle & 5 & .68 \\
\hline Apprentissages : fonctionnalité vs. développement personnel & 3 & .66 \\
\hline Rôle éducatif des pairs & 3 & .76 \\
\hline $\begin{array}{l}\text { Statut de l'enfant par rapport à l'adulte : égalitaire vs. } \\
\text { complémentaire }\end{array}$ & 4 & .50 \\
\hline Adaptation du rythme horaire de l'enfant & 3 & .61 \\
\hline Rythme de développement de l'enfant individuel vs. collectif & 3 & .79 \\
\hline $\begin{array}{l}\text { Représentation de l'intelligence de l'enfant : comportement social } \\
\text { vs. capacités de raisonnement }\end{array}$ & 4 & .85 \\
\hline Education selon le genre : égalitaire vs. différenciée & 3 & .75 \\
\hline Représentation de la personnalité de l'enfant : innée vs. acquise & 4 & .81 \\
\hline
\end{tabular}

Les répondants sont ainsi amenés à se prononcer sur des items tels que :

- Il faut apprendre à l'enfant seulement des choses correspondant à sa tranche d'âge (catégorie «rythme de développement de l'enfant individuel vs. collectif »)

- Pour réussir à réaliser une tâche, l'enfant doit d'abord recevoir des explications verbales (catégorie «stratégies éducatives : modèle instructionnel vs. participatif »)

- Il faut apprendre très tôt à l'enfant à maîtriser ses émotions (catégorie «attitudes attendues de l'enfant: maîtrise vs. spontanéité »)

Le questionnaire se termine par trois items à part portant sur l'évaluation du sentiment de convergence/divergence de conceptions de l'éducation avec l'enseignant-e, respectivement les parents, et deux items portant sur l'importance accordée à cette convergence/divergence. La passation des questionnaires s'est déroulée de décembre 2011 à juin 2012.

Trois types d'analyses quantitatives ont été menés :

- Des analyses de variance à partir des catégories d'items : analyses de variance entre les trois groupes de sujets, mais aussi par rapport aux autres variables individuelles. 
- Une analyse factorielle, sous forme d'une analyse en composantes principales, afin de dégager des dimensions d'ethnothéories et des modèles plus larges que nos catégories de départ. De nouvelles analyses de variances intergroupes et intragroupes ont ensuite été menées en prenant comme variables les dimensions obtenues par l'analyse en composantes principales.

- Une analyse de clusters, menée selon la méthode des Kmeans à partir des scores obtenus aux catégories d'items, afin de dégager une typologie d'ethnothéories et de regrouper les répondants en fonction de cette typologie et non des trois groupes définis a priori. La typologie obtenue et la répartition des répondants selon cette typologie ont été comparées avec la répartition intergroupe de notre échantillon (enseignantes - parents africains - parents portugais).

\section{Résultats}

\section{Analyses de variance}

Les analyses de variance relèvent des divergences d'ethnothéories significatives dans des domaines aussi divers que les stratégies éducatives, la constitution de l'environnement physique de l'enfant, l'usage de la récompense, les attitudes attendues de la part de l'enfant, le rôle développemental attribué à la situation d'échec, la conception des rôles éducatifs familiaux et des rôles éducatifs père-mère, la représentation des buts prioritaires de l'éducation, le rythme d'éducation conçu comme collectif ou individualisé, la différenciation ou non de l'éducation selon le sexe de l'enfant ou encore la représentation du rôle des pairs dans l'éducation. Les différences relevées entre parents et enseignantes par les analyses de variance sont confirmées par les réponses aux trois items en fin de questionnaire sondant le sentiment de divergence avec le partenaire d'éducation. Tant les parents (score moyen de 3.18 sur 5 pour les parents africains et de 2.7 pour les parents portugais) que les enseignantes (2.98) expriment un sentiment de divergence relativement important vis-à-vis des ethnothéories du partenaire de co-éducation.

Les résultats soulignent toutefois une complexité dans l'articulation entre les ethnothéories exprimées par les participants et les différentes variables analysées: d'une part enseignantes et parents ne divergent pas sur tous les domaines, certaines catégories témoignent d'une convergence 
d'opinions, à l'exemple de l'importance de la période du questionnement chez l'enfant ou de la représentation de l'usage de la sanction; d'autre part les divergences ne se situent pas systématiquement entre enseignantes et parents, de nombreux recoupements apparaissent, ainsi que des divergences intragroupes en fonction des autres variables prises en compte tant chez les enseignantes (âge, formation, fait d'être parent) que chez les parents (sexe, ancienneté d'arrivée en Suisse, formation, profession, nombre d'enfants, rang de l'enfant dans la fratrie).

Par exemple, une différence significative apparaît entre enseignantes en fonction de l'âge et de la formation (deux variables entre lesquelles existe une forte co-variance) quant à la représentation des rôles éducatifs du père et de la mère comme différenciés ou similaires : les enseignantes les plus jeunes et formées à la HEP défendent clairement une représentation égalitaire des rôles père-mère, rejoignant une majorité des parents portugais, tandis que les enseignantes plus âgées et formées à l'école normale prônent plutôt une différenciation des rôles, à l'image des parents africains. Autre illustration du côté des parents, ceux ayant suivi une formation universitaire ou exerçant une profession dans le tertiaire défendent davantage des stratégies éducatives axées sur le modèle participatif et se rapprochent ainsi des enseignantes.

Il serait donc fallacieux de penser que des divergences d'ethnothéories existent uniquement entre les groupes de départ de l'étude ou seulement entre enseignantes et parents. Les résultats témoignent d'une complexité que nous ne pouvons détailler entièrement ici. Les graphiques réunis dans la figure 1 résument les résultats des analyses de variance intergroupes pour les différentes catégories d'items, un astérisque signale les catégories dégageant des différences intergroupes significatives.

Figure 1 : Analyses de variance intergroupes selon les catégories d'items 


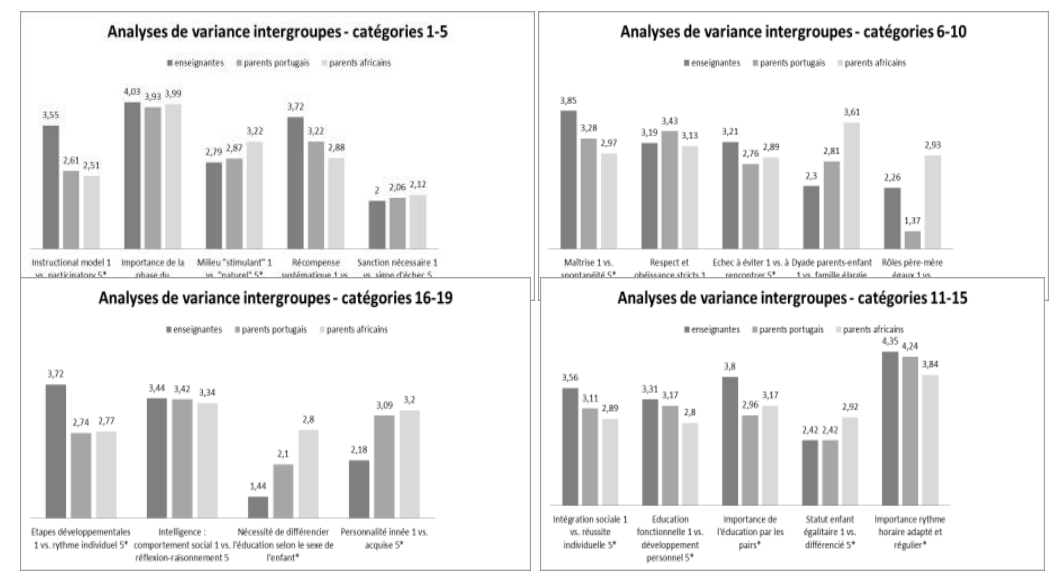

\section{Analyse en composantes principales}

L'analyse en composantes principales a pris pour variables les 19 catégories d'items du questionnaire, selon la méthode de rotation orthogonale. Les trois composantes sélectionnées l'ont été selon le critère de Cattell et elles expliquent $41.3 \%$ de la variance totale. Ce résultat certes assez bas, tout comme le score au test KMO (.646), s'expliquent en partie par la taille réduite de l'échantillon de notre étude. Les résultats apparaissent dans le tableau ci-dessous (Tableau 2), en ayant ôté les catégories dont la saturation n'était jugée suffisante dans aucune des composantes (seuil mis à .40).

Tableau 2 : Composantes de l'analyse en composantes principales

\begin{tabular}{|l|c|c|c|}
\hline ACP & Comp. 1 & Comp. 2 & Comp. 3 \\
\hline $\begin{array}{l}\text { Stratégies éducatives : modèle instructionnel } \\
\text { vs. participatif }\end{array}$ & .681 & & .408 \\
\hline Représentation de l'usage de la récompense & .536 & & \\
\hline Représentation de l'usage de la sanction & & & -.606 \\
\hline $\begin{array}{l}\text { Attitudes attendues de l'enfant : maîtrise vs. } \\
\text { spontanéité }\end{array}$ & .554 & -.405 & \\
\hline $\begin{array}{l}\text { Respect envers l'adulte : souple vs. rigide } \\
\text { Rôle développemental de la situation } \\
\text { d'échec }\end{array}$ & & -.511 & -.406 \\
\hline $\begin{array}{l}\text { Rôles éducatifs père-mère : différenciés vs. } \\
\text { similaires }\end{array}$ & .403 & .605 & .604 \\
\hline Buts de l'éducation : intégration sociale vs. & .663 & & \\
\hline
\end{tabular}




\begin{tabular}{|l|c|c|c|}
\hline réussite individuelle & & & \\
\hline $\begin{array}{l}\text { Apprentissages : fonctionnalité vs. } \\
\text { développement personnel }\end{array}$ & & & .589 \\
\hline Rôle éducatif des pairs & .701 & & \\
\hline $\begin{array}{l}\text { Rythme de développement de l'enfant } \\
\text { individuel vs. collectif }\end{array}$ & .721 & & \\
\hline Adaptation du rythme horaire de l'enfant & & & .613 \\
\hline $\begin{array}{l}\text { Représentation de l'intelligence de l'enfant : } \\
\text { comportement social vs. capacités de } \\
\text { raisonnement }\end{array}$ & & -.488 & \\
\hline $\begin{array}{l}\text { Statut de l'enfant : égalitaire vs. } \\
\text { complémentaire à l'adulte }\end{array}$ & & .477 & \\
\hline $\begin{array}{l}\text { Education selon le genre : égalitaire vs. } \\
\text { différenciée }\end{array}$ & & .581 & \\
\hline $\begin{array}{l}\text { Représentation de la personnalité de } \\
\text { l'enfant : innée vs. acquise }\end{array}$ & -.643 & & \\
\hline $\begin{array}{l}\text { TOTAL DE LA VARIANCE EXPLIQUÉE } \\
\text { PAR LA COMPOSANTE }\end{array}$ & $\mathbf{2 3 . 0 0 \%}$ & $\mathbf{9 . 6 0 \%}$ & $\mathbf{8 . 6 9 \%}$ \\
\hline
\end{tabular}

De l'analyse en composantes principales émergent trois dimensions. Nous commentons ici les deux premières de ces dimensions, les plus intéressantes dans l'analyse des positionnements intergroupes.

La première dimension, solide statistiquement ( $\alpha$ de Cronbach de .80 ), différencie un modèle éducatif participatif, horizontal et individuel (score 5 sur l'échelle de Likert), vers lequel tendent fortement les enseignantes, avec un score moyen de $3.72(S D=0.34)$, d'un modèle éducatif directif, vertical et collectif (score 1), auquel les deux groupes de parents accordent davantage d'importance que les enseignantes, avec un score moyen de $2.97(S D=0.66)$ pour les parents migrants portugais et de 2.86 $(S D=0.45)$ pour les parents migrants africains. Les résultats montrent une différence intergroupes clairement significative $(F=43.35, p<0.001)$. La différence est significative tant entre les enseignantes et les parents migrants portugais qu'entre les enseignantes et les parents migrants africains. Elle ne l'est pas entre les deux groupes de parents.

Le modèle éducatif participatif, horizontal et individuel met l'accent sur des stratégies éducatives impliquant la participation de l'enfant, une attente de spontanéité et d'initiative de la part de l'enfant, une représentation de l'éducation comme visant le développement personnel et individuel, une éducation à un rythme individuel et propre à chaque enfant. Le fait que les enseignantes tendent clairement vers ce modèle n'est pas une surprise, vu l'accent mis sur les méthodes actives et la pédagogie participative dans leur formation et dans leur culture 
professionnelle. Par contre, leur positionnement se distingue sensiblement de celui des parents qui se situent dans une position intermédiaire entre les deux pôles, accordant même une légère primauté au modèle éducatif directif, vertical et collectif. Ce modèle se caractérise par des stratégies éducatives axées sur une instruction verticale d'un agent éducatif actif envers un enfant la recevant de manière plutôt passive, l'attente d'attitudes de l'enfant d'abord marquées par la maîtrise et la discipline, une représentation de l'éducation comme à but premier d'intégration et de participation sociale, une éducation à un rythme commun en fonction d'étapes de développement dictées par l'âge. La variable de la formation des parents est intéressante dans l'analyse de cette première dimension : les parents de formation supérieure, qui sont tous des parents migrants africains dans l'échantillon, se distinguent significativement des autres parents en accordant sensiblement plus d'importance au modèle participatif, horizontal et individuel, même si cela demeure de manière bien moins tranchée que les enseignantes. Cette première dimension soulevée par l'analyse en composantes principales rappelle la dimension de l'individualisme-collectivisme décrite par Hofstede (2001), le modèle éducatif des enseignantes rejoignant le pôle individualiste de la dimension de Hofstede, le modèle éducatif des parents se positionnant plutôt sur le pôle collectiviste.

La deuxième dimension, intéressante dans l'analyse bien que moins solide statistiquement ( $\alpha$ de Cronbach de .43), distingue un modèle éducatif caractérisé par une forte différenciation des rôles et des statuts (rôles éducatifs du père et de la mère, différenciation de l'éducation selon le sexe de l'enfant, statut de l'enfant vis-à-vis de celui de l'adulte, ou encore attitudes attendues de la part de l'enfant), modèle correspondant au score 1 sur l'échelle de Likert, d'un modèle éducatif marqué par une faible différenciation de ces rôles et statuts, correspondant au score 5. Les trois groupes se positionnent de manière significativement différente $(F=4.69, p<0.05)$. Les enseignantes tendent le plus clairement vers le modèle à faible différenciation, avec un score moyen de $3.73(S D=0.42)$. Quant aux parents migrants africains, ils affichent le plus d'accord avec le modèle à forte différenciation, tout en restant dans une position relativement intermédiaire entre les deux pôles, avec un score moyen de 3.35 ( $S D=0.48)$. Les parents migrants portugais se situent entre deux, avec un score moyen de $3.53(S D=0.41)$. Les variables corrélées de la formation et de l'âge des enseignantes soulèvent une différence clairement significative sur cette dimension : les enseignantes plus jeunes et formées à la HEP tendent encore davantage vers le modèle à faible différenciation que les enseignantes plus âgées et formées à l'école normale, et se distinguent ainsi d'autant plus nettement du positionnement des deux groupes de parents. Nous pouvons là également 
faire un lien avec une dimension du modèle de Hofstede (2001), celle de la distance hiérarchique : le modèle éducatif à faible différenciation des rôles et des statuts, dont se réclament les enseignantes, est un modèle à faible distance hiérarchique, tandis que le modèle éducatif à forte différenciation des rôles et des statuts, davantage privilégié par les parents, correspond à un modèle à forte distance hiérarchique.

\section{Analyse de clusters}

L'analyse de clusters visait à 1) proposer une typologie des ethnothéories ; 2) en proposant cette typologie à partir des réponses individuelles plutôt qu'à partir de la variable des groupes de départ de l'étude, contrebalancer le risque de toute étude comparative intergroupes d'enfermer les sujets dans leur groupe de départ; 3) comparer les résultats obtenus avec ceux de l'analyse en composantes principales. $\mathrm{La}$ typologie que nous proposons suite à cette analyse de clusters distingue trois types d'ethnothéories. Elle confirme et synthétise les tendances observées dans les dimensions ressorties de l'analyse en composantes principales, mais en complexifiant l'articulation de ces deux dimensions et des catégories d'items, permettant une comparaison intergroupes plus fine.

Le tableau ci-dessous (Tableau 3) donne une vision synthétisée des caractéristiques permettant de différencier les trois types d'ethnothéories relativement aux catégories thématiques du questionnaire.

Tableau 3 : Typologie des ethnothéories issue de l'analyse de clusters

\begin{tabular}{|c|c|c|c|}
\hline Type d'ethnothéories & $\begin{array}{l}\text { Collectiviste- } \\
\text { directif genré }\end{array}$ & $\begin{array}{l}\text { Individualiste- } \\
\text { participatif non } \\
\text { genré }\end{array}$ & $\begin{array}{l}\text { Individualiste- } \\
\text { participatif } \\
\text { genré }\end{array}$ \\
\hline $\begin{array}{l}\text { Stratégies éducatives : } \\
\text { modèle instructionnel vs. } \\
\text { participatif }\end{array}$ & Directif + & Participatif + & Participatif ++ \\
\hline $\begin{array}{l}\text { Milieu éducatif familial } \\
\text { stimulant vs. naturel }\end{array}$ & Naturel + & $\begin{array}{c}\text { Construit-stimulant } \\
+\end{array}$ & $\begin{array}{c}\text { Construit-stimulant } \\
+\end{array}$ \\
\hline $\begin{array}{l}\text { Représentation de l'usage } \\
\text { de la récompense }\end{array}$ & Utile + & Contre-productif + & $\begin{array}{c}\text { Contre-productif } \\
++\end{array}$ \\
\hline $\begin{array}{l}\text { Attitudes attendues de } \\
\text { l'enfant : maîtrise vs. } \\
\text { spontanéité }\end{array}$ & $\begin{array}{c}\text { Maîtrise-Discipline } \\
+\end{array}$ & Spontanéité + & Spontanéité ++ \\
\hline $\begin{array}{l}\text { Rôle développemental de la } \\
\text { situation d'échec }\end{array}$ & $\begin{array}{c}\text { A éviter à l'enfant } \\
+\end{array}$ & Rôle formateur + & Rôle formateur + \\
\hline
\end{tabular}




\begin{tabular}{|c|c|c|c|}
\hline $\begin{array}{l}\text { Rôles éducatifs familiaux : } \\
\text { dyade enfant-parents vs. } \\
\text { famille élargie }\end{array}$ & Famille élargie + & $\begin{array}{l}\text { Dyade parents- } \\
\text { enfant }+\end{array}$ & $\begin{array}{l}\text { Dyade parents- } \\
\text { enfant }+\end{array}$ \\
\hline $\begin{array}{l}\text { Rôles éducatifs père-mère : } \\
\text { différenciés vs. similaires }\end{array}$ & Différenciés + & Egalitaires ++ & Différenciés ++ \\
\hline $\begin{array}{l}\text { Buts de l'éducation : } \\
\text { intégration sociale vs. } \\
\text { réussite individuelle }\end{array}$ & $\begin{array}{c}\text { Intégration sociale } \\
+\end{array}$ & $\begin{array}{c}\text { Réussite } \\
\text { individuelle + }\end{array}$ & $\begin{array}{c}\text { Réussite } \\
\text { individuelle + }\end{array}$ \\
\hline $\begin{array}{l}\text { Apprentissages : } \\
\text { fonctionnalité vs. } \\
\text { développement personnel }\end{array}$ & Fonctionnalité + & $\begin{array}{c}\text { Développement } \\
\text { personnel + }\end{array}$ & $\begin{array}{c}\text { Développement } \\
\text { personnel }+\end{array}$ \\
\hline Rôle éducatif des pairs & Secondaire + & Important + & Important ++ \\
\hline $\begin{array}{l}\text { Rythme de développement } \\
\text { de l'enfant individuel vs. } \\
\text { collectif }\end{array}$ & Collectif ++ & Individuel + & Individuel ++ \\
\hline $\begin{array}{l}\text { Education selon le genre : } \\
\text { égalitaire vs. différenciée }\end{array}$ & Différenciée + & Egalitaire ++ & Différenciée + \\
\hline $\begin{array}{l}\text { Représentation de la } \\
\text { personnalité de l'enfant : } \\
\text { innée vs. acquise }\end{array}$ & A acquérir + & Innée + & Innée ++ \\
\hline \multicolumn{4}{|l|}{ EFFECTIFS } \\
\hline ENSEIGNANTES & $\mathbf{0}$ & 24 & 7 \\
\hline PARENTS PORTUGAIS & 15 & 12 & $\mathbf{0}$ \\
\hline PARENTS AFRICAINS & 19 & 1 & 2 \\
\hline TOTAL & 34 & 37 & 9 \\
\hline
\end{tabular}

Les indications + / ++ / - / -- signalent la tendance plus ou moins forte vers le pôle d'ethnothéories en question.

Le premier type d'ethnothéories rassemble les caractéristiques suivantes : un accent net sur le modèle éducatif vertical et directif, une représentation du milieu davantage considéré comme permettant «naturellement » à l'enfant d'y faire ses apprentissages, une importance accordée à un usage assez systématique de la récompense, un accent mis sur les attitudes de maîtrise et de discipline de l'enfant autant que sur les attitudes de spontanéité et d'initiative, un rôle développemental moindre accordé à la situation d'échec rencontrée par l'enfant, qui doit donc plutôt être évitée, une éducation familiale comme étant en grande partie le rôle de la famille élargie, des rôles éducatifs entre père et mère en partie différenciés, des buts éducatifs d'abord en termes d'intégration sociale et de fonctionnalité, un rôle moindre accordé à l'éducation par les pairs, un rythme développemental commun et partagé entre enfants en fonction des âges, une éducation à différencier selon le sexe de l'enfant, et enfin une représentation de la personnalité comme façonnée par l'éducation et acquise. Nous le dénommons le type collectiviste-directif genré. 
Le type d'ethnothéories collectiviste-directif genré correspond à quasiment l'ensemble des parents africains (19/22). Chez trois répondants africains faisant exception, qui se retrouvent pour l'un dans le deuxième cluster et pour les deux autres dans le troisième, nous relevons une caractéristique commune, à savoir le niveau de formation: il s'agit de trois parents ayant suivi une formation universitaire. Ils se rapprochent davantage des ethnothéories des enseignantes, elles-mêmes réparties dans ces deuxième et troisième clusters. Il serait toutefois faux d'attribuer ces trois exceptions uniquement à la variable du niveau de formation, quatre autres parents africains ayant suivi une formation universitaire se rattachent quand même au premier cluster. Néanmoins, on peut dire que si dans l'ensemble les parents africains se distinguent nettement des enseignantes quant à leurs ethnothéories, le fait d'avoir suivi une formation supérieure peut diminuer ces divergences.

Concernant les parents portugais, quinze parents se rattachent au premier cluster, douze se rattachent au deuxième cluster. L'ancienneté d'arrivée en Suisse, le sexe du parent répondant, le nombre d'enfants ou encore le rang de l'enfant de la fratrie diffèrent parmi les parents portugais interrogés, mais aucune de ces variables ne montre de lien pertinent avec le positionnement dans le premier ou le second cluster. Quant aux variables du niveau de formation et du type de profession, elles témoignent d'une trop forte homogénéité chez les parents portugais pour y déceler quelque lien avec les types d'ethnothéories. Nous constatons toutefois que plus de la moitié des parents portugais $(15 / 27)$ se rapproche des ethnothéories de la majorité des parents africains, tandis qu'un peu moins de la moitié d'entre eux (12/27) se rapproche des ethnothéories d'une majorité d'enseignantes (24/31). De manière globale, l'analyse de clusters nous montre surtout qu'une nette majorité de parents interrogés (34/47), en se situant dans le premier cluster, affiche un type d'ethnothéories franchement différent de celui des enseignantes, qui se situent toutes dans le deuxième ou le troisième cluster.

Dans le second cluster, nous retrouvons donc, outre les douze parents portugais et un père africain, la majorité des enseignantes (24/31), avec les ethnothéories suivantes: un accent davantage mis sur le modèle éducatif participatif, une représentation du milieu de l'enfant comme devant être en partie construit de manière à être stimulant afin de favoriser son développement, un rôle plutôt négatif accordé à l'usage de la récompense, un accent mis sur les attitudes de spontanéité et d'initiative de l'enfant davantage que sur celles de maîtrise et de discipline, une importance supérieure au rôle développemental de l'échec, des rôles familiaux dans l'éducation avant tout réservés aux parents, une conception égalitaire dans la perception des rôles éducatifs mère-père, des objectifs axés sur la réussite individuelle et le 
développement personnel, un rôle accru attribué à l'éducation par les pairs, un rythme de développement et d'apprentissage individualisé en fonction du rythme propre à l'enfant, une non-différenciation de l'éducation selon le sexe de l'enfant, et enfin une représentation de la personnalité de l'enfant comme principalement innée et déjà existante. Nous l'appelons le type individualiste-participatif non-genré.

Le troisième et dernier cluster ne regroupe que neuf sujets (7 enseignantes et 2 parents africains), d'où quelques précautions à prendre dans l'interprétation. Il se caractérise par l'accent le plus nettement mis sur le modèle éducatif participatif, une représentation du milieu de l'enfant comme devant être encore davantage construit de manière à être stimulant pour son développement, une représentation de l'usage de la récompense comme très négatif d'un point de vue éducatif, un accent encore accru sur les attitudes de spontanéité et d'initiative de l'enfant plutôt que de maîtrise et de discipline, une représentation de l'échec comme ayant un certain rôle développemental, une éducation familiale comme étant le rôle des parents avant tout, une très forte différenciation des rôles éducatifs mère-père, des objectifs à l'éducation encore davantage en termes de réussite individuelle et de développement personnel, un rôle essentiel donné à l'éducation par les pairs, une individualisation très importante du rythme de développement en fonction de chaque enfant, une éducation à clairement différencier selon le sexe de l'enfant, et enfin une représentation de la personnalité de l'enfant comme essentiellement innée et déjà existante. Il s'agit du type individualiste-participatif genré.

Les sept enseignantes regroupées dans le type d'ethnothéories individualiste-participatif genré ont toutes été formées à l'école normale et appartiennent à la tranche plus âgée des enseignantes. Pour autant, toutes les enseignantes répondant à ces critères ne se retrouvent pas dans ce troisième cluster : seize d'entre elles se rattachent au deuxième type d'ethnothéories avec les enseignantes plus jeunes et formées en HEP. Ces variables corrélées de l'âge et de la formation ne doivent donc pas être interprétées de manière trop catégorique. Mais les sept enseignantes du troisième cluster semblent avoir une représentation plus ancienne de l'éducation, notamment marquée par une forte différenciation des rôles de la mère et du père, relativement aux représentations qui ont cours chez la majorité des enseignantes.

L'analyse de clusters propose ainsi une typologie des ethnothéories qui complète et relativise à la fois les résultats des analyses de variance et de l'analyse en composantes principales. D'un côté, la typologie nuance une vision des ethnothéories comme uniformes dans chacun des groupes de départ de l'échantillon. Même si des divergences intergroupes existent, 
les groupes de départ de l'étude ne sont pas homogènes quant à leurs ethnothéories, le positionnement des parents portugais notamment le montre bien. Les membres de chaque groupe ne partagent pas tous le même type d'ethnothéories, témoignant de la complémentarité entre dimensions collective (divergences intergroupes) et individuelle (divergences intragroupes) des ethnothéories. De l'autre côté, l'analyse de clusters souligne l'écart d'ethnothéories qui existe globalement entre enseignantes et parents : 34 parents sur 47 se retrouvent dans un type d'ethnothéories qui ne correspond à aucune des 31 enseignantes interrogées. La typologie révèle que la majorité des parents se situe dans un modèle éducatif plutôt directif, vertical et collectif, visant l'intégration sociale et la fonctionnalité, tandis que les enseignantes se retrouvent globalement dans un modèle plutôt participatif, horizontal et individuel, visant avant tout la réussite individuelle, avec pour une minorité non négligeable des enseignantes un positionnement assez extrême vers ce modèle.

\section{Discussion}

Nous souhaitions investiguer la variabilité interculturelle des ethnothéories de l'éducation. Si au vu des résultats de l'analyse intergroupes la variable de la culture du groupe apparaît comme un élément important dans le façonnement des ethnothéories, comme le postulent Super et Harkness (1986, 1996) et Dasen (1998), l'analyse de variables personnelles tant chez les enseignantes que chez les parents migrants révèle d'autres influences. Il serait à ce titre intéressant d'approfondir le facteur de la classe sociale, dont l'influence sur le rapport à l'école a été soulignée, notamment par Dubet et Martuccelli (1996) ou Périer (2005). Il serait ainsi pertinent d'étudier si l'accent mis par les parents de l'étude sur un modèle éducatif collectif n'est pas davantage lié à une attente envers l'école en termes d'égalisation des chances plutôt qu'à une différence culturelle. Il serait erroné de considérer la culture du groupe comme la seule explication des différences d'ethnothéories, dans une relation de causalité linéaire et homogénéisante entre culture et ethnothéories, contredite notamment par la diversité de types d'ethnothéories du groupe des parents portugais. Cependant, ne pas considérer du tout le lien entre la culture du groupe et la constitution des ethnothéories s'avérerait également un aveuglement. Les nombreuses divergences entre parents migrants portugais, parents migrants africains et enseignantes, mises en évidence tant dans les analyses de variance par catégories que dans les dimensions de l'analyse en composantes principales, soulignent le lien existant entre variable culturelle et constitution des ethnothéories. 
Il semble néanmoins surtout que ces divergences d'ethnothéories de l'éducation révèlent une culture scolaire diversement partagée par les répondants de notre étude. Malgré les divergences apparaissant parfois entre parents migrants africains et portugais, ou entre parents en fonction des variables intragroupes, les parents se positionnent souvent de manière assez similaire. Les divergences entre parents sont moins fréquentes et généralement moins prononcées que celles entre parents et enseignantes. Le constat vaut aussi pour les divergences observées à l'intérieur du groupe des enseignantes, qui sont de manière générale moindres que celles entre enseignantes et parents. Le fait que les divergences se situent le plus souvent entre une majorité de parents, indépendamment qu'ils soient des parents africains ou portugais, et l'ensemble des enseignantes, confirme le risque évoqué à interpréter cet écart de manière culturaliste en le mettant en lien uniquement avec une appartenance de groupe ethnique/nationale, et nous mène à dire qu'il y a avant tout une culture scolaire en termes de modèles éducatifs et d'ethnothéories, partagée par les enseignantes mais dont une majorité des parents interrogés se distancie. Nous retrouvons ainsi dans les réponses de notre échantillon de parents migrants ce que d'autres auteurs (notamment Périer, 2005 ; Delay, 2011) avaient relevé pour le contexte français ou suisse à propos des familles populaires. On peut imaginer les obstacles que cet écart entre culture scolaire et culture parentale/familiale va entraîner pour la mise en œuvre d'une coéducation à laquelle appelle l'injonction au partenariat.

Certains résultats à première vue surprenants étayent l'interprétation des divergences parents-enseignantes comme une différence entre culture parentale/familiale et culture scolaire, différence que l'on pourrait probablement aussi retrouver auprès de parents non migrants. Ainsi, on pouvait supposer que la variable de l'ancienneté d'arrivée en Suisse des parents serait significative, dans le sens d'une diminution des divergences corrélée à la durée de séjour, hypothèse qu'accréditerait le processus d'acculturation tel que décrit par Berry et al. (op. cit., 2002). Or l'influence de la variable de la durée de séjour n'apparaît absolument pas dans nos résultats. Lorsque divergence il y a entre les ethnothéories des parents migrants et celles des enseignantes, la variable de la durée de séjour ne montre pas de tendance à la diminution de cet écart à mesure que cette durée de séjour augmente. Concernant le sentiment de divergence des parents avec le partenaire d'éducation, on constate même une tendance à une légère augmentation corrélée à la durée de séjour. Ce résultat, certes de l'ordre d'une tendance et non d'une différence significative, témoigne du fait que d'autres variables, telles des expériences de collaboration précédentes autour d'un aîné, influencent certainement aussi les divergences d'ethnothéories et le sentiment de divergence. 
Si les résultats confirment notre hypothèse de départ postulant l'existence de divergences d'ethnothéories, le positionnement dans une majorité des cas clairement divergent entre parents et enseignantes dans leur ensemble nous interroge. Sans vouloir négliger les convergences qui existent également, ce constat questionne par rapport à la possibilité d'un réel partenariat école-familles. Les difficultés rencontrées par certains enfants à s'adapter au fonctionnement scolaire sont régulièrement mises sur le compte d'une absence de limites posées par les parents et d'une mentalité de l'enfant-roi qui aurait envahi de larges territoires de l'éducation familiale. Sans nier que cela puisse exister, les résultats nous mènent à relativiser la portée d'une telle explication. Les parents que nous avons interrogés semblent au contraire mettre davantage que les enseignantes l'accent sur un modèle éducatif directif et vertical, sur un statut inégalitaire de l'enfant vis-à-vis de l'adulte, ainsi que sur des attitudes attendues de l'enfant avant tout en termes de maitrise, de discipline et d'obéissance à l'adulte. La difficulté à s'adapter parfois au fonctionnement scolaire pourrait être davantage liée au désarroi de l'enfant à se situer face à un modèle éducatif différent de celui vécu dans le milieu familial, et non forcément à un problème de limites parentales.

Par cette hypothèse, nous ne souhaitons pas nous poser en pourfendeurs des modèles éducatifs de l'école, des méthodes actives et de la pédagogie participative, mais mettre l'accent sur une nécessaire explicitation des diverses conceptions de l'éducation, afin de pouvoir les négocier en vue d'un partenariat réel dans lequel chacun des acteurs puisse se retrouver de manière cohérente. L'injonction au partenariat, aujourd'hui omniprésente dans les décrets officiels, les textes de l'institution scolaire, les ouvrages des pédagogues, que disent souhaiter également les professionnels, appelle à la mise en œuvre d'une coéducation qui ne peut se réaliser sans une prise de conscience d'abord, et une négociation ensuite, des divergences de modèles éducatifs, en se basant sur une conception de la négociation définie dans une perspective communicationnelle: la communication, en l'occurrence entre l'école et la famille, est considérée comme un processus continu de co-construction et de négociation de sens entre les interactants (voir notamment Branco et Valsiner, 2004), avec pour résultat de cette négociation une interprétation nouvelle de l'objet. Cette interprétation nouvelle résulte de l'interaction, mais ne signifie pas nécessairement un accord comme le voudrait le sens commun de négociation. Toute négociation ne se déroule pas forcément sur un mode horizontal et égalitaire. Dans le contexte des relations entre l'école et les familles, on peut s'attendre même à ce qu'elle tende à se faire toujours sur un mode asymétrique et normatif. Mais le fait qu'une majorité de parents migrants n'adhère pas ou peu au modèle éducatif participatif et individuel prôné par l'école et les enseignants doit amener à considérer la 
mise en place d'un partenariat réel comme nécessitant davantage de prise de conscience des divergences de conceptions de l'éducation, puis d'explicitation, en vue de collaborer à trouver un terrain commun, dans le sens de la négociation de l'approche interculturelle de Cohen-Emerique (2011). Or, ce territoire commun, celui d'une culture éducative partagée dans lequel va pouvoir se construire la coéducation autour de l'enfant, est trop souvent pensé comme existant a priori.

Le travail de prise en compte des divergences d'ethnothéories ne peut être initié selon nous que par l'institution scolaire et ses représentants, les enseignants, car la relation école-familles s'inscrit dans une relation asymétrique de pouvoir où ce sont les enseignants qui se trouvent le plus souvent en position de force pour définir le mode selon lequel va se construire la relation avec les familles. Il est dès lors essentiel que les enseignants développent une pratique professionnelle qui prenne en compte l'existence des divergences de conceptions de l'éducation. Le partenariat n'implique évidemment pas que les enseignants doivent adhérer aux ethnothéories et aux pratiques éducatives des parents, mais il ne peut y avoir de réel partenariat sans que les enseignants soient conscients de l'existence de divergences à ce niveau et acquièrent, dans leur formation notamment, des aptitudes à expliciter, communiquer et échanger avec les parents sur les conceptions de l'éducation et les pratiques qui en découlent. Le constat des divergences d'ethnothéories signale le besoin pour la formation des enseignants de viser l'acquisition de pratiques de communication permettant de construire une relation basée sur une reconnaissance des différences dans laquelle pourra s'ancrer la coéducation autour de l'enfant.

\section{Perspectives}

Cette étude exploratoire et comparative a permis de mieux identifier et expliciter les divergences des ethnothéories de l'éducation détenues par des parents migrants et des enseignantes de l'école enfantine. Elle doit à présent ouvrir sur d'autres pistes de recherches, particulièrement dans deux directions : 1) élargir et diversifier la population, afin de consolider la validité des résultats et d'inclure d'autres groupes de parents, notamment non migrants, en vue de vérifier l'hypothèse qu'il existe des divergences d'ethnothéories entre enseignants et parents en général, et non seulement entre enseignants et parents migrants ; 2) investiguer, dans la démarche ethnographique évoquée en introduction, la manière dont parents et enseignants négocient ces divergences d'ethnothéories dans leurs interactions. Cette étude est une prémisse nécessaire mais non suffisante à des investigations ultérieures ayant pour objectif de comprendre comment peut se mettre en place une réelle coéducation, qui 
prenne en compte les ethnothéories des différents partenaires, particulièrement, mais non exclusivement, lorsque ceux-ci ont des références culturelles diverses.

\section{Références:}

Arm-Spring, C., Currat, F., Dumas, A., Fioravera Gomez, E., Fragnière, P., Gachoud, M.-L., Jordan, C., Meier, M., Rothen, B. et Viatte, L. (2008). A petits pas de grands projets. Outil pédagogique pour l'enseignement aux enfants de 4 à 7 ans. Le Mont-sur Lausanne : LEP Editions.

Berry, J. W., Poortinga, Y. H., Segall, M. H. et Dasen, P. R. (2002). Cross-cultural psychology. Research and applications (2è éd.). Cambridge : Cambridge University Press.

Bowler, J. (2005). Ecological and developmental issues in the practice of educational psychology. Kairaranga,6 (2), 49-54.

Branco, A. U. et Valsiner, J. (dir.). (2004). Communication and metacommunication in human development. Greenwich, CT : Information Age Publishers.

Bronfenbrenner, U. (1979). The ecology of human development. Experiments by nature and design. Cambridge (MA) and London : Harvard University Press.

Claes, M. et Comeau, J. (1996). L'école et la famille : deux mondes? Lien social et Politiques, 35, 75-85.

Cohen-Emerique, M. (2011). Pour une approche interculturelle en travail social. Théories et pratiques. Rennes : Presses de l'EHESP.

Comer, J. P. (1988). Educating poor minority children. Scientific American, 259, 42-48.

Conus, X. (2012). Les ethnothéories des parents et des enseignants. Etude comparative des ethnothéories d'enseignantes d'école enfantine et de parents migrants africains et portugais. (Mémoire de master non publié). Université de Fribourg, Suisse.

Dasen, P. R. (1998). Cadres théoriques en psychologie interculturelle. Dans J. G. Adair, D. Bélanger et K. L. Dion (dir.), Advances in psychological science. Volume 1: Social, personal and cultural aspects / Récents développements en psychologie scientifique. Volume 1: Aspects sociaux, personnels, culturels (pp. 205-227). Hove, East Sussex : Psychology Press Ltd., Publishers.

Dasen, P. R. (2002). Développement humain et éducation informelle. Dans P. R. Dasen et C. Perregaux (dir.), Pourquoi des approches interculturelles en sciences de l'éducation? (pp. 107-124). Bruxelles : De Boeck. 
Dasen, P. R. (2004). Education informelle et processus d'apprentissage. Dans A. Akkari et P. R. Dasen (dir.), Pédagogies et pédagogues du Sud (pp. 23-52). Paris: L'Harmattan.

Delay, C. (2011). Les classes populaires à l'école. La rencontre ambivalente entre deux cultures à légitimité inégale. Rennes: Presses Univ. de Rennes..

Doudin, P.-A., Lafortune, L., Pons, F. et Moreau, J. (2009). Le paradoxe de certaines mesures d'aide à l'intégration scolaire : quand l'aide mène à l'exclusion. Revue des sciences de l'éducation, 35 (1), 4153.

Dubet, F. (2001). Ecole, familles : le malentendu (2è éd.). Paris : Textuel.

Dubet, F. et Martuccelli, D. (1996). Les parents et l'école : classes populaires et classes moyennes. Lien social et Politiques, 35, 109121.

Dupeyron, J.-F. (2010). Nos idées sur l'enfance. Étude des représentations de l'enfance en Occident. Paris : L'Harmattan.

Epstein, J. L. (1991). Effects on student achievement of teachers' practices of parent involvement. Advances in Reading/Language Research, 5, 261-276.

Epstein, J. L. et Sanders, M. G. (2002). Family, school, and community partnerships. In M.H. Bornstein (dir.), Handbook of parenting Volume 5 Practical issues in parenting (pp. 407-438). Mahwah (New Jersey)-London : Lawrence Erlbaum Associates.

Epstein, J. L. et Sheldon, S. B. (2002). Present and accounted for : Improving student attendance through family and community involvement. The Journal of Educational Research, 95 (5), 308318.

Epstein, J. L. (2011). School, family and community partnerships. Preparing educators for improving schools (2è éd.). Boulder, CO : Westview Press.

Erny, P. (1987). L'enfant et son milieu en Afrique Noire. Paris : L'Harmattan.

Erny, P. (2001). Essai sur l'éducation en Afrique Noire. Paris: L'Harmattan.

Field, S., Kuczera, M. et Pont, B. (2007). En finir avec l'échec scolaire: Dix mesures pour une éducation équitable. Paris : OCDE.

Gayet, D. (1999). L'école contre les parents. Paris : INRP.

Hauser-Cram, P., Sirin, S. R. et Stipek, D. (2003). When teachers' and parents' values differ: Teachers' ratings of academic competence in children from low-income families. Journal of Educational Psychology, 95, 813-820. 
Hofstede, G. (2001). Culture's consequences : Comparing values, behaviors, institutions and organizations across nations (2è éd. révisée). Thousand Oaks, CA : Sage.

Hoover-Dempsey, K. V. et Sandler, H. M. (1997). Why do parents become involved in their children's education? Review of Educational Research, 67, 3-42.

Kim, Y. (2009). Minority parental involvement and school barriers: Moving the focus away from deficiencies of parents. Educational Research Review, 80-102.

Leanza, Y. (2003). Education, pédiatrie et cultures. Du sens de l'activité professionnelle pour des pédiatres dans leur travail de prévention auprès de familles migrantes. (Thèse de doctorat non publiée). Université de Genève, Suisse.

Mackiewicz, M.-P. (2010). Coéducation à l'école maternelle et engagement parental en Réseau d'Education Prioritaire. La revue internationale de l'éducation familiale, 28, 73-91.

Montandon, C. et Perrenoud, P. (1994). Entre parents et enseignants : un dialogue impossible? (2è éd.). Berne : Peter Lang.

Nsamenang, A. B. (2001). Perspective africaine sur le développement social : implications pour la recherche interculturelle de l'enfance et de l'adolescence. Dans C. Sabatier \& P. R. Dasen (dir.), Cultures, développement et éducation. Autres enfants, autres écoles (pp. 3952). Paris : L'Harmattan.

Nsamenang, A. B. (2005). The intersection of traditional African education with school learning. Dans L. Swartz, C. de la Rey et N. Duncan (dir.), Psychology: An introduction (pp. 327-337). Cape Town, South Africa : Oxford University Press.

Périer, P. (2005). École et familles populaires : sociologie d'un différend. Rennes : Presses Univ. de Rennes.

Poncelet, D. et Francis, V. (2010). Présentation du dossier “L'engagement parental dans la scolarité des enfants. Questions et enjeux." Revue internationale d'éducation familiale, 28, 9-20.

Porumbu, D. et Necşoi, D. V. (2013). Relationship between parental involvement/attitude and children's school achievements. Procedia - Social and Behavioral Sciences, 76, 706-710.

Prévôt, O. (2008). Attentes des familles à l'égard de l'école : une enquête auprès de 2492 parents. Dans G. Pithon, C. Asdih et S. J. Larivée (dir.), Construire une "communauté éducative". Un partenariat famille-école-association (pp. 37-50). Bruxelles : De Boeck Université.

Rayna, S. (dir.). (2010). Parents-professionnels : la coéducation en questions. Toulouse : Erès. 
Rimm-Kaufman, S. E. et Pianta, R. C. (2000). An ecological perspective on the transition to kindergarten: a theoretical framework to guide empirical research. Journal of Applied developmental psychology, 21, 491-511.

Reynolds, A. J. (1992). Comparing measures of parent involvement and their effects on academic achievement. Early Childhood Research Quarterly, 7, 441-462.

Super, C. M. et Harkness, S. (1986). The developmental niche : a conceptualization at the interface of child and culture. International Journal of Behavioral Development, 9 (4), 545-570.

Super, C. M. et Harkness, S. (1996). The cultural structuring of child development. Dans J. W. Berry, P. R. Dasen et T. S. Saraswathi (dir.), Handbook of cross-cultural psychology. Volume 2: Basic processes and human development (2è éd.) (pp. 1-39). Boston, MA : Allyn and Bacon. 